\title{
A Review of TQM and EHR Focused Quality
}

\author{
Mona Alghamdi ${ }^{1}$, Sara Alomari ${ }^{2}$, Mashael Althubaiti ${ }^{3}$, Azrillah Abdul Aziz ${ }^{4}$ \\ Information Systems Department, Faculty of computing \& Information Technology, King Abdulaziz University, \\ Jeddah, Saudi Arabia ${ }^{1,2,3}$
}

PhD, Information Systems Department, Faculty of computing \& Information Technology, King Abdulaziz University, Jeddah, Saudi Arabia ${ }^{4}$

\begin{abstract}
Information system plays a significant role related to heal thcare outcomes. It ensures that services are professionally delivered to individuals and populations by having updated knowledge and skills. It is important to realize that hospitals are looking for services of good quality to achieve a high level of customer satisfaction and loyalty. In this paper, we discuss Electronic Health Records Systems (EHR) as a type of these information systems that is widely used by healthcare providers. Firstly, we present the literature that emphasizes factors related to the adoption of an EHR system. Then, we define TQM and its principles and discuss the role of health information systems to fulfill these principles. Afterwards, we introduce its benefits and drawbacks. Finally, we investigate the effect of EHR implementation on healthcare quality. Furthermore, we highlight the literature's recommendations to achieve healthcare of high quality.
\end{abstract}

Keywords: Total Quality Management, TQM, EHR, electronic health records, healthcare quality, quality.

\section{INRODUCTION}

Nowadays, the information technologies help the organizations to achieve a high level of performance. It is important to realize that the quality of service has dramatically improved because of incorporating information systems and technologies. Particularly, the integration of IT into healthcare sector enables health care providers to manage health information securely and efficiently. It usually encompasses various electronic methods such as electronic health records (EHR) and computerized provider order entry.

In this paper, we focus on EHR systems as one of the most important technologies developed in health care. We introduce an overview of TQM and the role of health information systems to achieve TQM. Then, we discuss the definition of EHR, benefits and drawbacks, and how it influences the quality of care. Then, we investigate of literature to identify prominent metrics that affect the quality improvement. Moreover, to aggregate a set of recommendations and suggestions to optimize the quality of care using EHR systems.

\section{RESEARCH OBJECTIVE}

Total quality management concept has been used widely in many industries. Especially in healthcare organization, they aspire to gain a competitive advantage by applying the principles of TQM in all medical and administrative activities. For this purpose, they incorporated different health information systems such as electronic medical records (EMR) and electronic health records (EHR) to perform the activities efficiently.

Currently, the adoption of EHR helps health care providers to give best possible care. They will coordinate the care given more easily and efficiently. Also, they can involve patients by sharing information over the Internet and thus, patients can make decisions about their health care. The aim of this research is to give an insight into EHR systems and aggregate some recommendations and solutions to successfully utilize EHR systems to ensure high quality of care. Hence, it provides people of interest with a meaningful and structured knowledge regards to EHR systems.

\section{OVERVIEW OF TOTAL QUALITY MANAGEMENT}

TQM is a management philosophy used to raise the organization performance and deliver customer satisfaction. It could be a periodic process that ensures the quality and productivity of an organization where all members must participate to achieve its vision. It increases the customer satisfaction by applying set of guiding principles such as: meeting the needs of its customers, ensuring management involvement, applying continuous improvements and make the decision based on facts. Table 1 briefly describes TQM principles that lead to continuous improvement [1]. 
Vol. 4, Issue 5, May 2017

\begin{tabular}{|c|c|}
\hline Principle & Explanation \\
\hline Customer-focused & $\begin{array}{l}\text { Generally, the customer satisfaction can determine the level of quality. Regardless what an } \\
\text { organization does to achieve quality improvements, the customer decides whether these efforts } \\
\text { were beneficial. }\end{array}$ \\
\hline $\begin{array}{l}\text { Total employee } \\
\text { involvement }\end{array}$ & $\begin{array}{l}\text { All employees work together toward the same goal. They also contribute in managerial } \\
\text { decision making by performing normal business operations to accomplish the concept of } \\
\text { continuous improvement. }\end{array}$ \\
\hline Process-centered & $\begin{array}{l}\text { The processes have inputs and outputs. The outputs are what delivered to the customers. Thus, } \\
\text { the processes within the organization must be well-defined and continuously monitored to } \\
\text { immediately detect mistakes and errors. }\end{array}$ \\
\hline Integrated system & $\begin{array}{l}\text { To enhance the communication between the organization's departments, an integrated system } \\
\text { is required. It communicates the business processes information and performance } \\
\text { continuously to detect the level of improvement. }\end{array}$ \\
\hline $\begin{array}{l}\text { Continual } \\
\text { improvement }\end{array}$ & $\begin{array}{l}\text { The main goal of TQM is continual improvement. It drives the organization to find more } \\
\text { creative ways to be competitive. }\end{array}$ \\
\hline $\begin{array}{l}\text { Fact-based decision } \\
\text { making and strategic } \\
\text { approach }\end{array}$ & The decision making should be made based on facts about the business performance. \\
\hline
\end{tabular}

Table 1: TQM principles

\section{TQM and HEALTHCARE INFORMATION SYSTEMS}

For a healthcare organization, offering high quality services with affordable costs is a challenge. Patients are not willing to repeatedly fill forms when required since it increases the waiting time and leads to dissatisfaction. The following two sections will identify the relevant issues and highlight the role of information systems to accomplish TQM approach [2].

\section{A. TQM in healthcare}

As a matter of fact, healthcare organizations realized that TQM is vital. They seek to offer high quality services and diminish the waste. In addition, they strive to be more productive with lowers costs to satisfy their customers [2]. Due to the duality of authorities in health organizations, medical staff and administrative staff, TQM implementation may pose several problems. Therefore, the collaboration between these two authorities is essential and can be achieved with the use of information systems. The latter will enhance the communications between administrative staff and medical staff which, in turn, will assist in reducing conflicts. However, before the actual implementation of TQM in health organizations, we must ensure the support and the commitment of the top management. Then, we should realize the awareness of TQM by providing training sessions to educate the employees. A common cause of TQM failure is only using it to address problems and neglect the provided opportunities than can cause greatest return relative to the organizational objectives [2].

\section{B. The role of Hospital Information Systems (HIS)}

HIS has a major contribution in satisfying the principles of TQM. It is an integrated system that stores, retrieves, and communicates information about the components of a healthcare system. Indeed, HIS ought to help a healthcare organization to accomplish its goals and objectives Moreover, it assists the organizations to improve the quality of services and communications dramatically. There are several HIS systems that are designed to perform different healthcare operations and activities such as EHR and EMR. Each of which has its limitations and benefits. However, the use of theses HIS systems helps the management to save time and energy of carrying out day to day activities and spends them to focus on providing high quality of care [2].

\section{DEFINITION of EHR SYSTEMS}

Laramee et al. [2011] has defined an electronic health record as "a longitudinal electronic record of patient health information generated by one or more encounters in any care delivery setting". It is used to record the information like medical history in digital format [4]. In other words, EHR is archived electronic records that maintain information 
about an individuals' health status and health care. It provides different tools like clinical reminders and alerts. In addition, it analyzes accumulated data to be used in care management and research. In contrary to paper-based patient records, EHR allows the reader to organize, interpret and manipulate the data. Furthermore, all authorized personnel can access patients records immediately without a special permission or extra effort. Usually, computer-based records enhance the quality and ensure the validity of entered data. For instance, typographical can be disclosed by spell checkers and limited by input menus. Also, a computer-based system ensures the completeness of data since it can prompt users for additional information [5]. Many hospitals have adopted EHR, each of which has implemented a different approach. Their main goal is to realize a better performance in most clinical activities and procedures.

\section{CONCEPT of QUALITY in EHR SYSTEMS}

Currently, there is a debate if the EHR system can improve the quality of care. Some studies found that EHR-based practices lead to better quality of provided care. It coordinates the delivered care, enables authorized clinicians to view all patient data and reduces the possibility of encountering multiple physicians. Besides, it facilitates the transition of medical documents and provide physicians with recommendations based on the integrated knowledge resources. However, some studies reported that there are limitations of EHR systems on the enhancement of quality of care since there is no noticed improvement on patient outcomes has been accomplished. The use of EHR as a documentation mechanism rather than utilizing its capabilities of communications and decision-making might be the main reason. In addition, basic EHR systems can achieve more benefits than the advanced ones [6].

\section{EHR FUNCTIONALITIES}

In fact, EHR system is more than an electronic version of the paper records. It supports different functionalities like the communication as well as decision support functionalities.

\section{A. Integrated view of patient data}

It represents the primary goal of an EHR. It presents patient's data in a structured and an organized manner that is upto-date. Although, it poses many challenges because of the complexity and the variety of the data. In addition, no patient has a unique national identifier so that his/her data could be collected easily from different systems. Furthermore, even if patient's data are collected, the administrator of the EHR must ensure the compatibility of source data format with the EHR's. Otherwise, he must map the data format and coding to be acceptable to the EHR system [5].

B. Clinical decision support

A fundamental functionality in any healthcare system is clinical decision support. It presents clinicians with relevant data and information that are intelligently filtered and organized at appropriate times. The main goal is to enhance the level of health care by utilizing various tools to support decision-making in clinical workflow. These tools encompass reminders, alerts, documentation templates, reports and many other tools. CDS helps the system to print only related reminders before the scheduled visit using some rules and protocols [5].

C. Access to knowledge resources

Any EHR system must provide links to knowledge resources. Physicians access these resources to find answers to their queries and get knowledge about a particular patient situation. They usually access at the time of ordering online or reviewing results [5].

\section{Integrated communication and reporting support}

It is mandatory that healthcare organizations have the data they need when they needed. The EHR system, in turn, ensures not only the availability of data but the accuracy, integrity, and security of that data as well. The patient record should be one click away. Indeed, data must be communicated efficiently and effectively among clinic team members in order to assure the coordination of and well-timed care. Thus, a good EHR system owns tools to communicate data such as messages attached with the patient's record. These tools are critical when team members are separated geographically to promote networked communication that reaches all sites where care providers make decisions [5].

\section{A. Benefits of EHR}

\section{BENEFITS AND DRAWBACKS OF EHR}

Nowadays, most of the daily transactions are processed electronically as well as in medicine, where it is one of the richest information fields [6]. Many hospitals have adopted EHR to increase the level of care and data reliability. EHR helps to simplify the process of registering and managing the patient's data using a various interface. It manages patient's information including personal information, patient's histories, demographics, symptoms, laboratory test, and $\mathrm{x}$-ray results. It has automated all the tasks from entering prescriptions to ordering any drug or laboratory test. It has a positive impact on the services provided to the patients by reducing the delays of medical treatment and enhancing the quality of care by reducing the possible error. It helps the physician to make a decision and alerts them if there are any drug interactions or if the patient has an allergy of a certain type of food or medication. A study conducted by Dexter et 
al. [2001] reveals that 53\% of patients were eligible for preventive measures without physician admittance. In addition, it may reduce the averted costs by reducing record clerk cost, redundant use of test results and print out a thousand of patient's records. Patient's records are available anytime and anywhere, it could be reused and accessed immediately and remotely. EHR plays a very critical role in emergency cases by providing physicians with live-saving information [6]. Authorized users can access EHR record and share the information between many organizations involved in providing patients care and that will lead to eliminating redundant test papers, save time, and increase the efficiency.

The process of sharing information between EHR and organizations is called health information exchange that follows standards to ensure the information safety, consistency, and secure. It provides key information for emergency response, support research and provides effective and efficient care [7]. Information could be exchange in three forms of exchange: first, directed exchange, it is used to send patients information such as laboratory result to enable coordinator care; second, query-based exchange, it is used for unplanned care for example at emergency department when the physicians need to know about a patient medical history; and third, consumer-mediated exchange that patients can access and manage their health information online [8].

\section{Drawbacks of EHR}

Financial issues are one of the obstacles facing the implementation of EHR. Based on a research that surveyed all the American hospitals, the main barriers to implementing EHR are the capital requirements and high maintenance costs [9]. It requires purchasing software, hardware, converting paper materials into an electronic version and periodic maintenance. Also, training cost will be added; the system may be difficult to use for some people so training is essential. Moreover, the security is one of the concerns that limit EHR implementation. Attacking EHR can expose patient's data or make it not available for an unknown period of time [9].

Another challenge to implement EHR is the lack of standardization, which can impede to obtain the benefits of EHRs. Lack of standardization can make the physician hesitate to use other physician's data thus, all the terminology, technologies, apps and devices should follow a specific standard. The consistent data extracted from coherent health record are needed in making decision and assessing the quality of care [10].

\section{LITERATURE REVIEW}

Ludwick et al. [2009] have introduced a systematic review about health information system implementation from several sources. They have mentioned that quality of graphical user interface, system functionality and usability could positively affect the IS system implementation. Regards to EHR system implementation, Jha et al. [2009] have surveyed all the American Hospital and found that only 1.5\% hospitals have implemented the comprehensive electronic record due to the high maintenance cost and the capital requirement. Based on the study conducted by Elnahal et al. [2011], the level of hospital quality might be the reason.They reported the differences between high, intermediate and low-quality hospitals in adopting EHR functions.In addition, they defined Meaningful Use of EHR using a national survey data and took advantage of Hospital Quality Alliance to classify hospitals quality. They examined EHR adoption in hospitals of different levels of quality and tested if they met the Meaningful Use criteria. They found that high-quality hospitals were approximately satisfied all the Meaningful Use criteria such as reporting quality measures, realizing at least one decision support rule and exchanging clinical data. On the other hand, intermediate and lowquality hospitals still face challenges in adopting EHR systems. Consequently, they suggested that the level of quality of hospitals and EHR adoption may be associated. However, if a hospital implements the EHR system, it will gain superior benefits. As stated in [Zlabek et al., 2011], the incorporating the Computerized Provider Order Entry with inpatient EHR resulted in a rapid reduction of cost of care and enhanced the safety of hospitals. They conducted a comparison between the cost and safety metrics before and after the implementation of inpatient EHR with CPOE. While Welch et al. [2007] have proved the benefits by assessing the cost and quality of care before and after EHR implementation. They considered the rate of guidelines adherence as a measure of quality. The use of EHR helps to adhere the guidelines more faster. Regards to the cost, it was difficult to decide the impact since there were no tools that accurately measure the long-term costs.

Nevertheless, the implementation of EHR is not sufficient to achieve the quality of care as expressed by Baron [2007]. $\mathrm{He}$ insists to adopt EHR system based on widespread standards and to enter data into the system in a structured manner. One way to enhance the quality of data is to strengthen the security of the EHR system. Terry et al. [2007] assert that enhancing the security of EHR can limit medical errors and offer a platform for patients to be involved. Thus, the quality of care is achieved. It is important to realize the data quality since it has a direct impact on the decision-making. Some errors may prevent the organization to meet its performance goals. Ward et al. [2015] have discussed the level of data errors in EHR that can influence the accuracy of operational performance metrics. They utilized a 3-months data set and used Monte Carlo simulation to present types of data errors. They figured out that small data errors hinder 


\section{International Advanced Research Journal in Science, Engineering and Technology} ISO 3297:2007 Certified

Vol. 4, Issue 5, May 2017

determining the performance goals accurately. Therefore, They suggested best data management practices to ensure measuring true performance. In order to meet the performance goals perfectly, we should consider the time aspect when implementing EHR systems. Poissant et al. [2005] highlighted that time efficiency is a significant goal of implementing EHR systems. They gathered 23 papers based on specific criteria and organized them according to a methodology they have used. The combination of results of the studies has led to a conclusion that the documentation time is increased regards to the physicians. In contrast, the use of EHR by nurses causes a reduction in the documentation time. However, Linder et al. [2012] investigated the impact of the documentation style on the quality. They negotiated three styles which are dictation, structured and free text. The results of the study show that dictation style is the worst quality comparing to other styles. Physicians who used EHR-based structured style deliver a higher quality of care. Hence, the authors suggest increasing the usability of EHR system so physicians become willing to use it frequently because they usually face difficulties when using EHR technology as illustrated by Walsh [2004]. He explained how clinicians work and gave recommendations on EHR system design, accordingly. In addition, he examined how the use of the system might affect the patient care. Furthermore, some researchers investigated the time duration of using EHR and the quality of outcomes [20]. They examined how frequent these functions are used among EHR users and inspected if this has an association with the duration of EHR.They focused on major EHR functions which are decision support, data management, health information and data, order entry management and communication and connectivity. The results showed that there is no relationship between them, and the duration of using EHR has zero effect on the delivered quality of care.

\section{CONCLUSION}

In this paper, we explained how Total Quality Management concept plays a critical role in health information system. Implementing TQM principles can raise the organization performance and deliver customer satisfaction. To successfully adopt the quality in EHR; top management must be committed and involved in the process and training sessions must be provided to the employees. In addition, we found that EHR quality helps healthcare providers to deliver quality care efficiently in shortest time and reduces the delays of patient's medical treatment. It limits the medical errors regarding the penmanship prescriptions and billing. Moreover, it reduces the documentation time and provides patient data management through a simple user interface.

\section{REFERENCES}

[1] Khadijah Mohammed, Bashayer Ali Alotibie, Azrilah Abdulaziz: “Total Quality Management in Saudi Higher Education”, International Journal of Computer Applications, pp. 6-12, 2016.

[2] B. Khorramshahgol, J. Al-Barmil, R. W. Stallings: "TQM in hospitals and the role of information systems in providing quality services", Proceedings for Operating Research and the Management Sciences, pp. 196-199, 1995.

[3] Ann S Laramee, Marcia Bosek, Christina A Kasprisin, and Terry Powers-Phaneuf. Learning from within to ensure a successful implementation of an electronic health record. CIN: Computers, Informatics, Nursing, 29(8):468-477, 2011.

[4] Dave A Ludwick and John Doucette. Adopting electronic medical records in primary care: lessons learned from health information systems implementation experience in seven countries. International journal of medical informatics, 78(1):22-31, 2009.

[5] Paul C Tang and Clement J McDonald. Electronic health record systems. In Biomedical informatics, pages 447-475. Springer,2006.

[6] Doug Morrison Swati Yanamadala: "Electronic Health Records and Quality of Care”, Medicine, 2016.

[7] Paul R Dexter, Susan Perkins, J Marc Overhage, Kati Maharry, Richard B Kohler, and Clement J McDonald. A computerized reminder system to increase the use of preventive care for hospitalized patients. New England Journal of Medicine, 345(13):965-970, 2001.

[8] Claudia Williams, Farzad Mostashari, Kory Mertz, Emily Hogin, and Parmeeth Atwal. From the office of the national coordinator: the strategy for advancing the exchange of health information. Health affairs, 31(3):527-536, 2012.

[9] Ashish K Jha, Catherine M DesRoches, Eric G Campbell, Karen Donelan, Sowmya R Rao, Timothy G Ferris, Alexandra Shields, Sara Rosenbaum, and David Blumenthal. Use of electronic health records in us hospitals. New England Journal of Medicine, 360(16):1628-1638, 2009.

[10] Marilyn Skrocki. Standardization needs for effective interoperability. 2013.

[11] Shereef M Elnahal, Karen E Joynt, Steffanie J Bristol, and Ashish K Jha. Electronic health record functions differ between best and worst hospitals. The American journal of managed care, 17(4):e121, 2011.

[12] Jonathan A Zlabek, Jared W Wickus, and Michelle A Mathiason. Early cost and safety benefits of an inpatient electronic health record. Journal of the American Medical Informatics Association, 18(2):169-172, 2011.

[13] W Pete Welch, Dawn Bazarko, Kimberly Ritten, Yo Burgess, Robert Harmon, and Lewis G Sandy. Electronic health records in four community physician practices: impact on quality and cost of care. Journal of the American Medical Informatics Association, 14(3):320-328, 2007.

[14] Richard J Baron. Quality improvement with an electronic health record: achievable, but not automatic. Annals of internal medicine, 147(8):549-552, 2007.

[15] Nicholas P Terry and Leslie P Francis. Ensuring the privacy and confidentiality of electronic health records. U. Ill. L. Rev. , page 681, 2007.

[16] Michael J Ward, Wesley H Self, and Craig M Froehle. Effects of common data errors in electronic health records on emergency department operational performance metrics: A monte carlo simulation. Academic Emergency Medicine, 22(9):1085-1092, 2015.

[17] Lise Poissant, Jennifer Pereira, Robyn Tamblyn, and Yuko Kawasumi. The impact of electronic health records on time efficiency of physicians and nurses: a systematic review. Journal of the American Medical Informatics Association,12(5):505-516, 2005. [18] Jeffrey A Linder, Jeffrey L Schnipper, and Blackford Middleton. Method of electronic health record documentation and quality of primary care. Journal of the American Medical Informatics Association, 19(6):1019-1024, 2012.

[19] Stephen H Walsh. The clinician's perspective on electronic health records and how they can affect patient care. Bmj, 328(7449):1184-1187, 2004.

[20] Li Zhou, Christine S Soran, Chelsea A Jenter, Lynn A Volk, E John Orav, David W Bates, and Steven R Simon. The relationship between electronic health record use and quality of care over time. Journal of the American Medical Informatics Association, 16(4):457-464, 2009. 